OPEN ACCESS

Edited by:

Yuanping Zhou,

Southern Medical University, China

Reviewed by:

Ila Pant,

Icahn School of Medicine at Mount

Sinai, United States

Guohong Deng,

Army Medical University, China

${ }^{*}$ Correspondence:

Jun Liu

dr_liujun1967@126.com

Specialty section:

This article was submitted to

Gastrointestinal Cancers,

a section of the journal

Frontiers in Oncology

Received: 16 June 2021 Accepted: 11 October 2021

Published: 10 November 2021

Citation:

Yang Y, Li G, LU Z, LiU Y,

Kong J and Liu J (2021)

Progression of Prothrombin

Induced by Vitamin K Absence-II

in Hepatocellular Carcinoma.

Front. Oncol. 11:726213.

doi: 10.3389/fonc. 2021.726213

\section{Progression of Prothrombin Induced by Vitamin K Absence-II in Hepatocellular Carcinoma}

\author{
Yang Yang ${ }^{1,2}$, Guangbing $L^{2}{ }^{2}$, Ziwen $L u^{1,2}$, Yong Liu ${ }^{1}$, Junjie Kong ${ }^{2}$ and Jun Liu ${ }^{1,2 *}$ \\ ${ }^{1}$ Department of Liver Transplantation and Hepatobiliary Surgery, Shandong Provincial Hospital, Cheeloo College of Medicine, \\ Shandong University, Jinan, China, ${ }^{2}$ Department of Liver Transplantation and Hepatobiliary Surgery, Shandong Provincial \\ Hospital Affiliated to Shandong First Medical University, Jinan, China
}

Hepatocellular carcinoma ( $\mathrm{HCC})$ is the fifth most common cancer and the third leading cause of cancer-related death worldwide. Due to the lack of efficient tools for early detection, asymptomatic HCC patients are diagnosed at an advanced stage, leading to a poor prognosis. To improve survival, serum biomarker prothrombin induced by vitamin $\mathrm{K}$ absence-II (PIVKA-II) was under investigation. PIVKA-II is an abnormal protein produced in HCC. The coagulation function was insufficient due to the lack of Gla residues. Elevated PIVKA-II was associated with bad tumor behavior in terms of proliferation, metastasis, and invasion. Three major signaling pathways were proposed to clarify the mechanism. With the advantages including affordability, minimal invasiveness, convenience, and efficiency, PIVKA-II could improve HCC management consisting of four aspects. First, PIVKA-II was an effective and dynamic tool for improving HCC surveillance in high-risk population. Changes in the serum levels of PIVKA-II provided valuable molecular alteration information before imaging discovery. Second, PIVKA-II offered a complementary approach for HCC early detection. Compared to traditional diagnostic approaches, the combination of PIVKA-II and other biomarkers had better performance. Third, PIVKA-II was an indicator for the assessment of response to treatment in HCC. Preoperative assessment was for selecting personalized therapy, and postoperative measurement was for assessing treatment efficacy. Fourth, PIVKA-II was considered as a prognostic predictor for HCC. Patients with elevated PIVKA-II were more likely to develop microvascular invasion, metastasis, and recurrence.

Keywords: hepatocellular carcinoma, prothrombin induced by vitamin K absence-II, biomarker, diagnosis, prognosis

\section{INTRODUCTION}

Hepatocellular carcinoma (HCC) is the fifth most common cancer and the third leading cause of cancer-related death worldwide $(1,2)$. The disease burden of HCC is increasing annually $(3)$. The etiology of HCC is highly complex and diverse. Roughly $85 \%$ of HCC derives from cirrhosis secondary to hepatitis B virus (HBV) and hepatitis C virus (HCV) (4-6). The process in 
thecontext of chronic liver diseases takes several years or decades (7-9). Despite advancements in systemic therapies including transarterial chemoembolization (TACE), liver transplantation, targeted therapy, and immunotherapy, surgical resection is the optimal treatment for HCC. As asymptomatic HCC patients are diagnosed at an advanced stage, they are not amenable to curative surgery. The 5-year overall survival was only $15 \%(10-12)$. Thus, early detection was crucial to improving prognosis.

Currently, early detection in HCC relies on surveillance in high-risk population. Patients with chronic viral hepatitis and other chronic liver diseases such as alcoholic liver disease, nonalcoholic fatty liver disease (NAFLD), and non-alcoholic steatohepatitis (NASH) are recognized as a high-risk group of HCC. Abdominal ultrasound (US) every 6 months for populations at high risk was recommended by guidelines (13, 14). However, US is an operator-dependent technique and has limitations in the differential diagnosis. With remarkable advantages, noninvasive and cost-effective serum biomarkers were proposed. Serum $\alpha$-fetoprotein (AFP) is a wellestablished biomarker for HCC screening. But the diagnostic accuracy is suboptimal with sensitivity at $62.4 \%$ (15-18). To address the limitations of AFP in conjunction with abdominal ultrasound, investigators proposed a novel serum biomarker for HCC surveillance and early detection-prothrombin induced by vitamin $\mathrm{K}$ absence-II (PIVKA-II).

PIVKA-II, also known as Des-gamma-carboxy prothrombin (DCP), is an abnormal protein produced in hepatocellular carcinoma (19). The coagulation function was insufficient due to the lack of Gla residues. Elevated PIVKA-II in HCC was associated with bad tumor behavior in terms of proliferation, metastasis, and invasion. Liebman et al. first presented PIVKA-II for HCC diagnosis in $1984(20,21)$. They found that PIVKA-II was significantly elevated in patients with HCC compared to patients with chronic liver diseases. Since then, the wide implementation of PIVKA-II had shown great performance in HCC early detection. Multiple studies suggested that PIVKA-II could facilitate HCC surveillance in high-risk population, especially for dynamic monitoring (22). Meanwhile, PIVKA-II was proposed as a potential tool for prognostic prediction in terms of vascular invasion, metastasis, and recurrence. PIVKA-II was also used in therapeutic response assessment during treatments. The efficacy of treatments including hepatic resection, liver transplantation, radiofrequency ablation, and interventional therapy could be evaluated (23-26). In this context, PIVKA-II helped guide the personalized therapies. It is worth noting that the implementation of PIVKA-II in other tumors in clinical practice has been demonstrated such as primary gastric cancer, pancreatic adenocarcinoma, etc $(27,28)$.

This review focused on the recent progression of PIVKA-II in HCC in the past years. The literature summarized the structure and function of PIVKA-II and clinical relevance for surveillance in high-risk population, early detection, assessment of response to therapy, and prognostic prediction in HCC (Figure 1).

\section{STRUCTURE AND SIGNALING PATHWAYS OF PIVKA-II}

\section{The Structural Characteristics and Biological Origin of PIVKA-II}

PIVKA-II, known as Des-gamma-carboxy prothrombin (DCP), is a prothrombin precursor produced in HCC. Prothrombin in hepatocytes is constituted from amino acid residues, composed of three different structural domains, fragment 1 , fragment 2 , and a protease domain. There are 10 glutamic acid (Glu) residues at positions $6,7,14,16,19,20,25,26,29$, and 32 in the $\mathrm{N}$-terminal domain (29). Normal prothrombin in hepatocyte converted the 10 Glu residues into $\gamma$-carboxylated glutamic acids termed as Gla in the protease domain $(30,31)$. The posttranslational carboxylation of the prothrombin precursor depended on vitamin K-dependent $\gamma$-glutamyl carboxylase. HCC cells are unable to carboxylate all of Glu residues due to carboxylase deficiency (32). The remaining Glu residues are called des- $\gamma$ carboxy prothrombin. PIVKA-II contains less than 10 Gla residues, resulting in a deficiency of thrombogen synthesis (33). Hemker et al. first observed the biosynthesis of PIVKA-II indirectly in 1963 (34). Then Nilehn and Ganrotin validated it directly (35). Stenflo et al. (36) explained the differences between PIVKA-II and normal prothrombin. They found that PIVKA-II was a kind of prothrombin lacking modified residues with glutamic acid, failing to bind with calcium ions. Due to the absence of structural integrity, PIVKA-II was unable to play roles in the activation of coagulation. It was not proposed as an efficient serum biomarker for HCC diagnosis until 1984 (37). Liebman et al. reported that PIVKA-II was significantly increased in $91 \%$ of HCC patients without normal sufficient coagulant activity (38). And the number of Gla residues and their positions determined the biological activity of the tumor $(39,40)$. If the number is less than 5, the liver disease has a higher tendency to be malignant $(41,42)$. The functions caused by different position deletion in the initiation and progression of HCC need further validations. Numerous studies evaluated its values in predicting prognosis-associated pathological parameters and assessing treatment efficacy in HCC. Recently, PIVKA-II has shown great prospects in other tumor detection and prediction such as primary gastric cancer, pancreatic adenocarcinoma, etc $(43,44)$.

\section{Signaling Pathways of PIVKA-II}

PIVKA-II was a potential autologous growth stimulator for HCC proliferation. The biological process was enhanced by regulating cell proliferation, extracellular matrix (ECM) synthesis, and angiogenesis. Hepatocyte growth factor (HGF) was associated with HCC biological process binding to membrane-spanning receptor tyrosine kinase (c-Met). PIVKA-II could also bind to cMet by phosphorylation similar to hepatocyte growth factor (HGF) (45). Then the elevated conjugants activated the downstream signals including JAK1-signal transducers and activators of transcription (STAT3), extracellular signalregulated kinase (ERK1/2)-mitogen-activated protein kinase 


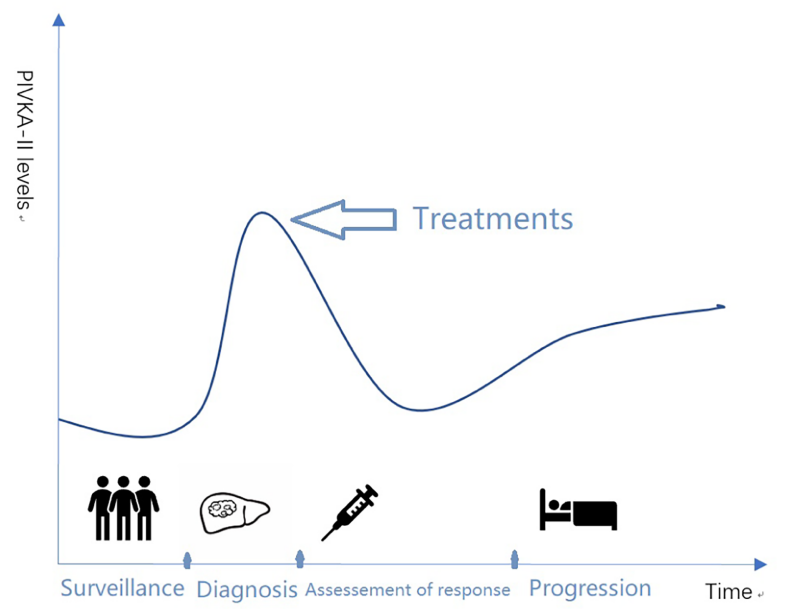

FIGURE 1 | Relative levels of PIVKA-II for HCC management.

(MAPK), and kinase insert domain receptor-phospholipase C- $\gamma$-mitogen-activated protein kinase (KDR-PLC- $\gamma$-MAPK). The activated signaling pathways promoted HCC progression in terms of proliferation, metastasis, and invasion (46-48) (Figure 2). The key signaling pathway for HCC proliferation was c-Met-JAK-STAT3 $(49,50)$. The signaling pathway was activated through the overexpression of PIVKA-II/c-Met that enhanced the DNA synthesis and cell proliferation in HCC (51-53).

Elevated PIVKA-II was associated with bad tumor behavior in terms of metastasis and invasion, leading to a poor prognosis. Many studies have attempted to clarify the mechanism. From current evidence, PIVKA-II could stimulate EGFR phosphorylation, activating Raf serine/threonine kinases. Perhaps PIVKA-II induced the Raf- MEK 1/2 -ERK1/2-MAPK signaling pathway. Then MMP-2 and MMP-9 increased in the context of regulation. With the degradation of the extracellular matrix, MMPs facilitated endothelial cells for matrigel invasion. In addition, the signaling pathway promoted the mitotic process by stimulating mitogens, growth factors, and cytokines (54). This process played a great role on proliferation, metastasis, and invasion in HCC (52).

Angiogenesis was associated with the continuous growth of tumor tissues and tumor progression. Angiogenic factors such as fibroblast growth factor (FGF), transforming growth factor- $\alpha$ (TGF- $\alpha$ ), and basic fibroblast growth factor (bFGF) stimulated proliferation and endothelial cell invasion. PIVKA-II was also considered an angiogenic factor. PIVKA-II not only promoted the secretion of other angiogenic factors in HCC cells but also induced KDR-PLC- $\gamma$-MAPK signaling pathway in vascular endothelial cells, leading to extracellular matrix degradation and cell migration $(55,56)$.

\section{CLINICAL RELEVANCE OF PIVKA-II IN HCC}

\section{Surveillance For HCC in High-Risk Population}

Surveillance in high-risk populations is an effective measure for HCC early detection. Chronic viral hepatitis-related HCC accounts for $80 \%$ of HCC. Patients with virus-related cirrhosis were recommended for HCC surveillance every 6 months (57). Recent studies indicated that patients with other chronic liver diseases such as alcoholic liver disease, non-alcoholic fatty liver disease (NAFLD), and non-alcoholic steatohepatitis (NASH) could also benefit from surveillance $(58,59)$. A 6-monthly abdominal ultrasonography examination and AFP level measurement are widely implemented for HCC surveillance. The US is an essential first imaging modality, a noninvasive tool despite the sensitivity being 63\% (60). Serum biomarker AFP was available in HCC surveillance. However, the levels of AFP were normal in 35-40\% HCC patients, causing false negatives. Thus, EASL excluded AFP measurement from the guideline due to its insufficient diagnostic accuracy $(61,62)$. In this context,

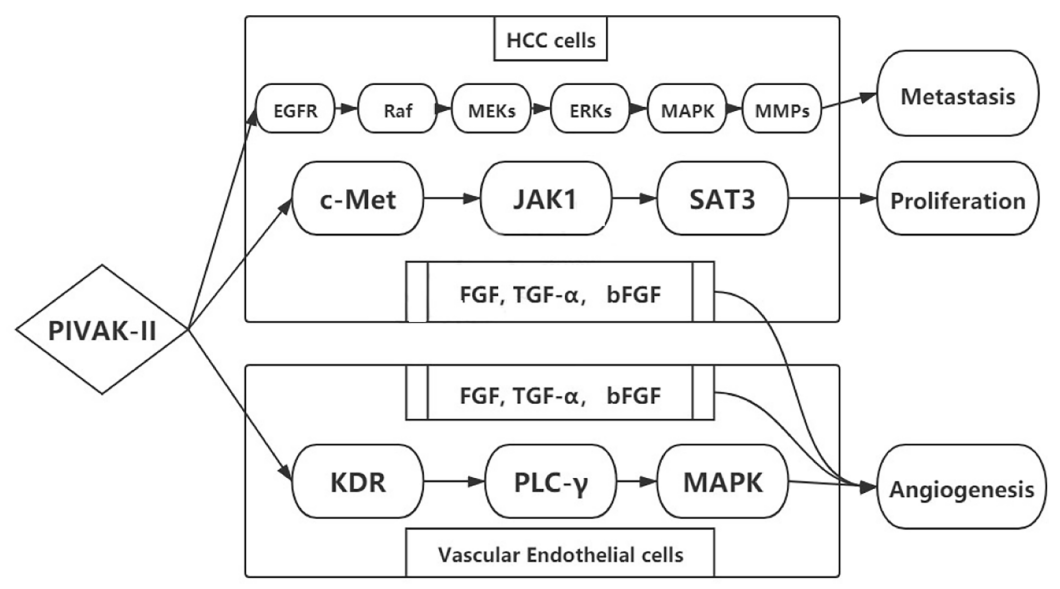

FIGURE 2 | Mechanism of PIVKA-II in HCC progression. 
serum biomarker PIVKA-II was emerging as a complementary tool for HCC surveillance. Currently, the measurement of combined PIVKA-II and AFP was endorsed in Japan Clinical Practice Guidelines for HCC surveillance (63).

Regarding PIVKA-II as a biomarker, it was promising for HCC surveillance. A meta-analysis evaluated the efficacy of PIVKA-II in 459 cases with hepatitis B virus from June 2016 to March 2018 (64). The pooled sensitivity, specificity, and area under the curve (AUC) were 0.71, 0.93, and 0.91, respectively (64). The study demonstrated the better performance of PIVKAII in hepatitis B virus-related HCC surveillance. However, due to potential underlying diseases, PIVKA-II alone was not on the point of implementation.

Compared to traditional tools for HCC surveillance, combined PIVKA-II and AFP enhanced the accuracy. PIVKA-II appeared to be more effective in the differential diagnosis between regenerative/dysplastic and neoplastic nodules. A study evaluated the value of HCC surveillance in 90 patients with cirrhotic liver nodules. The result demonstrated that PIVKA-II combined with AFP increased the specificity to 94\%, while it decreased the sensitivity. PIVKA-II was more likely to reflect molecular alterations preceding tumor development for cirrhotic patients before imaging discovery. Thus, the combination of PIVKA-II and AFP was a highly promising tool for cirrhotic patient surveillance. Caviglia et al. compared the efficacy of AFP, PIVKA-II, and glypican-3 (GPC-3) for HCC surveillance. The study consisted of 200 patients with viral hepatitis-related cirrhosis and a follow-up period of 36 months. A total of 86 patients who developed HCC had elevated PIVKA-II compared to those still HCC-free. PIVKA-II was the only serum biomarker that could indicate the increased risk of HCC in cirrhotic patients (65).

Considering available repeated peripheral blood samples, changes of PIVKA-II could be applied to personalized dynamic monitoring $(66,67)$. In 2020, a case-control study assessed the levels of PIVKA-II in patients with cirrhosis during the 12month observation period. The results showed that PIVKA-II had an increasing trend over time during the progress to HCC. The value at the latest time-point in HCC-developed patients and HCC-free patients were 66 and $32 \mathrm{mAU} / \mathrm{ml}$, respectively $(p<0.001)(68)$. The study suggested that PIVKA-II could serve as an alternative and dynamic tool for HCC surveillance and made it possible to tailor personalized surveillance (64).

\section{Early Detection in HCC}

Given the difficulty in early detection in HCC, only $20-30 \%$ of asymptomatic patients had the chance of radical resection (69). Serum PIVKA-II has received much attention as a biomarker for earlier detection to achieve long-term survival in HCC. The wellestablished biomarker AFP evaluated in many studies had high proportions of false positives. The novel biomarker PIVKA-II had better diagnostic performance with higher specificity in HCC early detection despite the conditions including vitamin $\mathrm{K}$ deficiency, malnutrition, drug (e.g., warfarin), and obstructive jaundice $(64,70,71)$. In addition, the mechanism of PIVKA-II and AFP was independent. The concentrations of two biomarkers in the same patient were unrelated to each other (72). In this context, PIVKA-II was under investigation as a promising complementary tool $(73,74)$.

Multiple studies supported that the combination of PIVKA-II and AFP had better diagnostic efficacy in HCC with considerable $67 \%$ sensitivity and $100 \%$ specificity $(75,76)$ (Table 1). Hemken et al. reported that the levels of AFP and PIVKA-II both elevated in patients with HCC, while the combination was superior to any biomarker alone in early detection (area under the curve 0.85 , 95\% CI 0.81-0.88) (80). Such findings were similar in a validated study (81). In the study, a diagnostic nomogram based on biomarkers for patients with chronic liver diseases has been constructed to improve diagnostic accuracy. The reliability was confirmed by a multicenter validation study. The combination of AFP and PIVKA-II had good performance in HCC early detection especially for differential diagnosis [AUC, 0.922 (95\% CI, $0.908-0.935$ ), sensitivity $88.3 \%$, and specificity $85.1 \%$ for the training cohort; 0.902 (95\% CI, 0.875-0.929), sensitivity $87.8 \%$, and specificity $81.0 \%$, for the validation cohort] (81). Recently, studies described the GALAD score based on gender, age, AFP, AFP-L3, and PIVKA-II. The score had the superior efficacy (sensitivity $93.3 \%$, specificity $85.6 \%$ ) regardless of imaging findings $(78,82)$. Indeed, PIVKA-II could be considered a complementary biomarker to others for early detection in HCC.

However, whether combined biomarkers could improve the accuracy was still a point of contention. In 2020, contradictory results were described that combined PIVKA-II and AFP was not better at identifying HCC at the early stage (83).

A needle biopsy was the golden standard for confirmed diagnosis in HCC. Its widespread use was restricted due to the

TABLE 1 | Studies of diagnostic performance of PIVKA-II and its combinations in HCC.

\begin{tabular}{|c|c|c|c|c|c|c|c|}
\hline \multirow[t]{2}{*}{ Author } & \multirow[t]{2}{*}{ Year } & \multirow[t]{2}{*}{$\mathbf{N}$} & \multirow[t]{2}{*}{ Biomarkers } & \multirow[t]{2}{*}{ Cut-off value } & \multicolumn{3}{|c|}{ Diagnostic Performance } \\
\hline & & & & & Sensitivity (\%) & Specificity (\%) & AUROC \\
\hline Yu R. et al. (77) & 2016 & $45 / 183$ & PIVKA-II+AFP & PIVKA- $\|=32 \mathrm{mAU} / \mathrm{ml}$ AFP $=5.0 \mathrm{ng} / \mathrm{ml}$ & 88.0 & 85.2 & 0.886 \\
\hline Lim TS et al. (78) & 2016 & $361 / 637$ & PIVKA-II+AFP+AFP-L3 & PIVKA- $\|=40 \mathrm{mAU} / \mathrm{ml}$ AFP=20ng $/ \mathrm{mL}$ AFP-L3 $=5 \%$ & 87 & 60.1 & 0.877 \\
\hline \multirow[t]{2}{*}{ Sultanik P. et al. (79) } & 2017 & 46/162 & PIVKA-II & PIVKA-II=73mAU/ml & 74 & 85 & 0.89 \\
\hline & & & PIVKA-II+AFP & PIVKA-\|=128mAU/ml AFP=20ng/mL & 87 & 76 & A \\
\hline \multirow[t]{2}{*}{ Loglio A. et al. (76) } & 2020 & $64 / 212$ & PIVKA-II & PIVKA- $\|=48 \mathrm{mAU} / \mathrm{ml}$ & 64 & 91 & 0,776 \\
\hline & & & PIVKA-II+AFP & PIVKA- $\|=48 \mathrm{mAU} / \mathrm{ml}$ AFP=4.2ng $/ \mathrm{mL}$ & 80 & 86 & 0.830 \\
\hline \multirow[t]{2}{*}{ Caviglia G. et al. (65) } & 2020 & $149 / 349$ & PIVKA-II & PIVKA- $\|=73 \mathrm{mAU} / \mathrm{ml}$ & 68 & 84 & 0.790 \\
\hline & & & PIVKA-II+AFP & PIVKA- $\|=73 \mathrm{mAU} / \mathrm{ml}$ AFP=9.7ng/mL & 70 & 86 & 0.822 \\
\hline \multirow[t]{2}{*}{ Feng $\mathrm{H}$. et al. (70) } & 2021 & $168 / 321$ & PIVKA-II & PIVKA- $\|=35.6 \mathrm{mAU} / \mathrm{ml}$ & 83.93 & 91.50 & 0.90 \\
\hline & & & PIVKA-II+AFP & PIVKA-\|=35.6mAU/ml AFP=17.76ng $/ \mathrm{mL}$ & 87.50 & 92.50 & 0.94 \\
\hline
\end{tabular}


risk of complications such as bleeding, infection, and needle tract seeding (84). Imaging examinations were minimally invasive and effective. However, it depended on the expertise of the physician and technological devices, resulting in difficulties in confirmed diagnosis (85). Affordable, minimally invasive, convenient, and efficient serum biomarker PIVKA-II was highly anticipated as a complementary tool to liquid biopsy for confirmed diagnosis in $\operatorname{HCC}(77,86,87)$.

\section{Assessment of Response to Therapy in HCC}

Currently, available treatments for HCC included hepatectomy, transarterial chemoembolization (TACE), radiofrequency ablation (RFA), liver transplantation, targeted therapy, and immunotherapy $(88,89)$. With the advancements of HCC therapeutic strategies and growing awareness of molecular pathogenesis, accurate treatment evaluation based on a molecular level was crucial for selecting optimal treatment strategy. PIVKA-II was considered to have an influence on the assessment of response to therapy for HCC patients in two ways.

On one hand, PIVKA-II may help in preoperative evaluation. PIVKA-II levels identified good candidates who will benefit from liver transplants (LTs). Current Milan criteria (MC) for LT were defined as a single tumor $\leq 5 \mathrm{~cm}$ or the tumor number was less than 3 , tumor size $\leq 3 \mathrm{~cm}$, and no major vascular invasion. The criteria were so strict that fewer patients gain. Expended criteria based on serum biomarkers have been proposed. Studies reported that preoperative elevated PIVKA-II was an independent predictor of recurrence and metastasis after LT. Patients with levels of AFP+PIVKA-II $\leq 300 \mathrm{mAU} / \mathrm{ml}$ could benefit from liver transplant. In addition, a meta-analysis confirmed that elevated PIVKA-II was associated with a fivefold increased risk for HCC recurrence after liver transplant (90-92).

Compared to hepatic resection, radiofrequency ablation (RFA) had the following advantages: minimal invasiveness, definite efficacy, high safety, and fast recovery. However, when serum levels of PIVKA-II $>100 \mathrm{mAU} / \mathrm{ml}$, patients who received hepatic resection had a better prognosis compared to patients with local ablation therapy (24). He et al. established a scoring system for predicting tumor recurrence after RFA. The scoring system suggested that PIVKA-II $>40 \mathrm{mAU} / \mathrm{ml}$ was an independent risk factor of early recurrence after RFA in $\operatorname{HCC}(93,94)$. In this context, PIVKA-II could provide useful information to improve outcome prediction for HCC management.

On the other hand, early changes of PIVKA-II levels were detected as an indicator for monitoring response to treatments in HCC. PIVKA-II contributed to establishing a standard for assessing the efficacy of hepatectomy. Kim et al. evaluated the trends of PIVKA-II in 184 patients with curative surgery. They found that 94 patients (51.1\%) with elevated PIVKA-II levels postoperatively developed intrahepatic recurrence during the follow-up period. Results showed that postoperative PIVKAII $>40 \mathrm{mAU} / \mathrm{m}$ indicated poor therapeutic efficacy of hepatectomy (95). The assessment would also apply to TACE. Reduced PIVKA-II after TACE indicated good efficacy. Levels of PIVKA-II in HCC patients with TACE were evaluated. Patients with no PIVKA-II response had shorter survival (7.1 vs 75.8 months) and a high risk of cancer-related death $(\mathrm{P}=0.02)(95)$. Another study suggested that advanced patients with a reduction of PIVKA-II $>50 \%$ after TACE had better survival compared to patients with no response (67.0 vs. 19.8 months) $(96,97)$. Similar findings were subsequently observed in patients with curative ablation that postoperatively elevated PIVKA-II levels were associated with poor prognosis (24). Overall, the measurement of serum PIVKA-II should be integrated into clinical practice for response assessment.

\section{Prognostic Prediction in HCC}

$\mathrm{HCC}$ has a poor prognosis with a high degree of malignancy. The overall survival varies from 3 to over 60 months $(98,99)$. The prognosis was associated with clinical staging in terms of tumor burden (number and size) and vascular invasion, which were independent prognostic risk factors for HCC patients (79). However, current staging systems for prognostic prediction in HCC were based on imaging or pathologic results and rarely included serum biomarkers. Serum biomarkers AFP and PIVKA-II have been approved as predictors for HCC prognosis. A novel staging system including remnant liver function (such as bilirubin and albumin) and serum biomarkers (such as PIVKA-II, AFP, and AFP-L3) had been established to predict survival regardless of the diverse etiology of HCC. The system showed good prognostic prediction efficacy with real-time and dynamic monitoring (100-103).

The preoperative macrovascular invasion could be detected by imaging examination, while microvascular invasion (MVI) relied on postoperative pathological results. How to detect MVI preoperatively is constantly in question. As an angiogenic factor, serum PIVKA-II was proposed as a predictor for MVI in HCC. It was not clear what the optimal cutoff was. Many investigators have assessed the cutoff values (Table 2) (24, 104-107). Pote et al. represented PIVKA-II $90 \mathrm{mAU} / \mathrm{ml}$ as an independent predictor of MVI (HR 3.5; 95\% CI 1.08-11.8; p=0.043) (109). Ryu et al. reported that the cutoff value was $55 \mathrm{mAU} / \mathrm{ml}$ (OR, 5.50; 95\% CI, 2.09-15.4; $\mathrm{P}<0.001$ ) (107). In addition, serum PIVKA-II could be used as a supplement to imaging findings in the prediction of macrovascular invasion. Wu et al. evaluated preoperative levels of PIVKA-II and postoperative pathological results through 31 patients with macrovascular invasion. Results suggested that PIVKA-II $>166$ $\mathrm{mAU} / \mathrm{ml}$ was a significant predictor of macrovascular invasion (OR 2.997; 95\% CI, 1.217-7.381; $\mathrm{P}=0.017$ ) in HCC (106).

Elevated PIVKA-II was associated with aggressive clinicopathological features. Although total tumor burden may be associated with high levels of PIVKA-II, some studies reported that HCC tumors with elevated PIVKA-II had a higher malignancy grade and were potentially more prone to metastasize $(110,111)$. Suk-Won Suh et al. reported that the level of PIVKA-II $\geq 800 \mathrm{mAU} / \mathrm{ml}$ could predict micrometastases and poor prognosis $(\mathrm{HR}=5.166$; 95\% CI, 1.031-25.897) (112). In addition, serum PIVKA-II levels were related to tumor size, tumor cell differentiation, and BCLC staging $(\mathrm{P}<0.05)(108)$. Si et al. reported that the serum levels of PIVKA-II were significantly different in well, moderate, or poor differentiation HCC (108). 
TABLE 2 | Cutoff value of PIVKA-II as an independent predictor in HCC vascular invasion.

\begin{tabular}{|c|c|c|c|c|c|}
\hline Author & Year & $\mathbf{N}$ & Cut-off Value & OR & P Value \\
\hline Masuda T. et al. (24) & 2016 & $46 / 217$ & $100 \mathrm{mAU} / \mathrm{ml}$ & 2.61 & $P=0.02$ \\
\hline Wang X. et al. (104) & 2017 & $14 / 59$ & $32 \mathrm{mAU} / \mathrm{ml}$ & 1.003 & $P=0.047$ \\
\hline Okamura Y. et al. (105) & 2018 & $76 / 425$ & $55 \mathrm{mAU} / \mathrm{ml}$ & 9.74 & $P<0.001$ \\
\hline Wu J. et al. (106) & 2018 & $31 / 91$ & 166mAU/ml & 2.997 & $P=0.017$ \\
\hline Ryu T. et al. (107) & 2019 & $49 / 111$ & $55 \mathrm{mAU} / \mathrm{ml}$ & 5.50 & $P<0.001$ \\
\hline
\end{tabular}

Patients with elevated PIVKA-II were characterized by a high relapse rate and short overall survival. Early and aggressive recurrence occurred after curative therapies (113). Elevated PIVKA-II may reflect the potential of HCC progression regardless of optimal treatment. According to previous literature, serum levels of PIVKA-II $>217 \mathrm{mAu} / \mathrm{ml}$ was an independent risk factor of early HCC recurrence $(\mathrm{p}=0.0001)$ $(114,115)$. Feng Gao et al. reported that the cutoff value of PIVKA-II was $445 \mathrm{mAU} / \mathrm{ml}(\mathrm{RR}=2.307,95 \% \mathrm{CI}: 1.132-4.703$, $\mathrm{P}=0.021$ ) (33). However, the association between low PIVKA-II levels and longer survival was weak (116). Since liver dysfunction affected prognosis, a multicenter study investigated disease-free survival and overall survival of HCC patients with Child-Pugh classes B and C. Patients with PIVKA-II $<90 \mathrm{mAu} / \mathrm{ml}$ had better 5-year survival. Elevated PIVKA-II levels were the superior prognostic predictor for HCC recurrence (117).

\section{CONCLUSIONS AND FUTURE PROSPECTIVE}

PIVKA-II was a prothrombin precursor produced in HCC. The coagulation function was insufficient due to the lack of Gla residues. Liebman et al. first described PIVKA-II as a biomarker for HCC diagnosis in 1984. Much evidence indicated that elevated PIVKA-II in HCC was associated with malignant tumor behavior in terms of proliferation, migration, and angiogenesis. Three major signaling pathways were proposed to clarify the mechanism. The key signaling pathway for HCC growth was PIVKA-II-c-Met-JAK-STAT3. Raf-MEK1/ 2-ERK1/2-MAPK-MMPs were associated with HCC migration and invasion. PIVKA-II was considered as an angiogenic factor through KDR-PLC- $\gamma$-MAPK, highly expressed in both HCC cells and vascular endothelial cells. The improved understanding of PIVKA-II at the molecular level might provide potential druggable targets for HCC treatment (118).

HCC is a malignant tumor with high morbidity and mortality. Since radical resection remains the only potentially curative treatment for HCC, early detection is essential. Over the past decades, US and biomarker AFP have been widely used for HCC management with suboptimal accuracy. Given the difficulties in early detection, patients with HCC had poor prognosis. To prolong survival, serum biomarker PIVKA-II was proposed for early detection and prognostic prediction in HCC.

PIVKA-II was a potential serum biomarker for HCC. With the advantages including affordability, minimal invasiveness, convenience, and efficiency, PIVKA-II could improve HCC management in four aspects. First, PIVKA-II was an effective and dynamic tool for improving HCC surveillance in high-risk populations. Changes of serum levels of PIVKA-II offered valuable molecular alteration information before imaging discovery. Second, PIVKA-II offered a complementary approach for HCC early detection. Compared to traditional diagnostic approaches, the combination of PIVKA-II and other biomarkers had better performance. Third, PIVKA-II was an indicator for the assessment of response to treatment in HCC. Preoperative assessment was for selecting personalized therapy, and postoperative measurement was for assessing treatment efficacy. Fourth, PIVKA-II was considered as a prognostic predictor for HCC. Patients with elevated PIVKA-II were more likely to develop microvascular invasion, metastasis, and recurrence.

Despite the progressions of PIVKA-II in HCC, some points need to be addressed. The first is whether PIVKA-II associated with other biomarkers could significantly improve the efficacy for HCC management. Second, what is the optimal cutoff value? Third, what could be the mechanism of PIVKA-II in HCC progression? Further studies are required.

Although serum PIVKA-II was not widely accepted in clinical practice, it is highly anticipated as a biomarker for surveillance, early detection, treatment monitoring, and prognostic prediction in HCC.

\section{AUTHOR CONTRIBUTIONS}

All authors helped to perform the research; YY and JL were involved in the conception and design; GL assisted in searching the latest literature about the role and application of PIVKA-II; JK assisted in language polishing; ZL assisted in creation and modification of figures and charts; YY and YL were involved in the drafting of the paper or revising it critically for intellectual content; JL were involved in the final approval of the version to be published. All authors agreed to be accountable for all aspects of the work.

\section{FUNDING}

This work was fund by the National Natural Science Foundation of China (General Program), No.81770646.

\section{ACKNOWLEDGMENTS}

We thank the members of Department of Hepatobiliary Surgery and Center of Organ Transplantation, Shandong Provincial Hospital for guiding the direction and supporting. 


\section{REFERENCES}

1. Siegel R, Miller K, Jemal A. Cancer Statistics, 2016. CA: Cancer J Clin (2016) 66:7-30. doi: 10.3322/caac. 21332

2. Fattovich G, Stroffolini T, Zagni I, Donato F. Hepatocellular Carcinoma in Cirrhosis: Incidence and Risk Factors. Gastroenterology (2004) 127:S35-50. doi: 10.1053/j.gastro.2004.09.014

3. Estes C, Anstee Q, Arias-Loste M, Bantel H, Bellentani S, Caballeria J, et al. Modeling NAFLD Disease Burden in China, France, Germany, Italy, Japan, Spain, United Kingdom, and United States for the Period 2016-2030. J Hepatol (2018) 69:896-904. doi: 10.1016/j.jhep.2018.05.036

4. Adnan F, Khan N, Iqbal A, Ali I, Petruzziello A, Sabatino R, et al. Interleukin-6 Polymorphisms in HCC Patients Chronically Infected With HCV. Infect Agents Cancer (2020) 15:21. doi: 10.1186/s13027-020-00285-9

5. Lin L, Yan L, Liu Y, Qu C, Ni J, Li H. The Burden and Trends of Primary Liver Cancer Caused by Specific Etiologies From 1990 to 2017 at the Global, Regional, National, Age, and Sex Level Results From the Global Burden of Disease Study 2017. Liver Cancer (2020) 9:563-82. doi: 10.1159/000508568

6. Parkin DM, Bray F, Ferlay J, Pisani P. Global Cancer Statistics, 2002. CA Cancer J Clin (2005) 55:74-108. doi: 10.3322/canjclin.55.2.74

7. Romeo R, Petruzziello A, Pecheur EI, Facchetti F, Perbellini R, Galmozzi E, et al. Hepatitis Delta Virus and Hepatocellular Carcinoma: An Update. Epidemiol infection (2018) 146:1612-8. doi: 10.1017/S0950268818001942

8. Busch K, Thimme R. Natural History of Chronic Hepatitis B Virus Infection. Med Microbiol Immunol (2015) 204:5-10. doi: 10.1007/s00430014-0369-7

9. Di Bisceglie AM. Hepatitis B and Hepatocellular Carcinoma. Hepatology (2009) 49:S56-60. doi: 10.1002/hep.22962

10. Li S, Yao J, Xie M, Liu Y, Zheng M. Exosomal miRNAs in Hepatocellular Carcinoma Development and Clinical Responses. J Hematol Oncol (2018) 11:54. doi: 10.1186/s13045-018-0579-3

11. Fujiki M, Takada Y, Ogura Y, Oike F, Kaido T, Teramukai S, et al. Significance of Des-Gamma-Carboxy Prothrombin in Selection Criteria for Living Donor Liver Transplantation for Hepatocellular Carcinoma. Am J Transplant Off J Am Soc Transplant Am Soc Transplant Surgeons (2009) 9:2362-71. doi: 10.1111/j.1600-6143.2009.02783.x

12. Zhang BH, Yang BH, Tang ZY. Randomized Controlled Trial of Screening for Hepatocellular Carcinoma. J Cancer Res Clin Oncol (2004) 130:417-22. doi: 10.1007/s00432-004-0552-0

13. Chen X, Zhao Y, Gao Y, Qi Y, Du J. Outcomes in Hepatocellular Carcinoma Patients Undergoing Sorafenib Treatment: Toxicities, Cellular Oxidative Stress, Treatment Adherence, and Quality of Life: Erratum. Anti-Cancer Drugs (2021) 32:345-64. doi: 10.1097/CAD.0000000000001029

14. EASL-EORTC Clinical Practice Guidelines: Management of Hepatocellular Carcinoma. J Hepatol (2012) 56:908-43. doi: 10.1016/j.jhep.2011.12.001

15. EASL Clinical Practice Guidelines: Management of Hepatocellular Carcinoma. J Hepatol (2018) 69:182-236. doi: 10.1016/j.jhep.2018.03.019

16. Yin L, He N, Chen C, Zhang N, Lin Y, Xia Q. Identification of Novel BloodBased HCC-Specific Diagnostic Biomarkers for Human Hepatocellular Carcinoma. Artif Cells Nanomedicine Biotechnol (2019) 47:1908-16. doi: 10.1080/21691401.2019.1613421

17. Kim H, Kim K, Choi J, Lee JM, Lee CH, Kang TW, et al. Comparison of Biannual Ultrasonography and Annual Non-Contrast Liver Magnetic Resonance Imaging as Surveillance Tools for Hepatocellular Carcinoma in Patients With Liver Cirrhosis (MAGNUS-HCC): A Study Protocol. BMC Cancer (2017) 17:877. doi: 10.1186/s12885-017-3819-y

18. Giannini EGMS, Borgonovo EG, Marenco S, Borgonovo G, Savarino V, Farinati F, Del Poggio P, et al. Alpha-Fetoprotein has No Prognostic Role in Small Hepatocellular Carcinoma Identified During Surveillance in Compensated Cirrhosis. Hepatol Res Off J Japan Soc Hepatol (2012) 56 (4):1371-9. doi: 10.1002/hep.25814

19. Cui S, Yu X, Qu X. Roles and Signaling Pathways of Des- $\gamma$ Carboxyprothrombin in the Progression of Hepatocellular Carcinoma. Cancer Invest (2016) 34:459-64. doi: 10.1080/07357907.2016.1227445

20. Saitta C, Raffa G, Alibrandi A, Brancatelli S, Lombardo D, Tripodi G, et al. PIVKA-II Is a Useful Tool for Diagnostic Characterization of UltrasoundDetected Liver Nodules in Cirrhotic Patients. Medicine (2017) 96:e7266. doi: 10.1097/MD.0000000000007266
21. Li C, Zhang Z, Zhang P, Liu J. Diagnostic Accuracy of Des-Gamma-Carboxy Prothrombin Versus $\alpha$-Fetoprotein for Hepatocellular Carcinoma: A Systematic Review. Hepatol Res (2014) 44:E11-25. doi: 10.1111/hepr.12201

22. Gao J, Song P. Combination of Triple Biomarkers AFP, AFP-L3, and PIVAKII for Early Detection of Hepatocellular Carcinoma in China: Expectation. Drug Discov Ther (2017) 11:168-9. doi: 10.5582/ddt.2017.01036

23. Gao F, Zhu H, Zhu YB, Shan QN, Ling Q, Wei XY, et al. Predictive Value of Tumor Markers in Patients With Recurrent Hepatocellular Carcinoma in Different Vascular Invasion Pattern. Hepatobiliary Pancreatic Dis Int HBPD Int (2016) 15:371-7. doi: 10.1016/S1499-3872(16)60095-4

24. Masuda T, Beppu T, Okabe H, Nitta H, Imai K, Hayashi H, et al. Predictive Factors of Pathological Vascular Invasion in Hepatocellular Carcinoma Within $3 \mathrm{Cm}$ and Three Nodules Without Radiological Vascular Invasion. Hepatol Res Off J Japan Soc Hepatol (2016) 46:985-91. doi: 10.1111/ hepr.12637

25. Zhang D, Liu Z, Yin X, Qi X, Lu B, Liu Y, et al. Prognostic Value of PIVKA-II in Hepatocellular Carcinoma Patients Receiving Curative Ablation: A Systematic Review and Meta-Analysis. Int J Biol Markers (2018) 33:26674. doi: 10.1177/1724600818760234

26. Poté N, Cauchy F, Albuquerque M, Voitot H, Belghiti J, Castera L, et al. Performance of PIVKA-II for Early Hepatocellular Carcinoma Diagnosis and Prediction of Microvascular Invasion. J Hepatol (2015) 62:848-54. doi: 10.1016/j.jhep.2014.11.005

27. Kemik AS, Kemik O, Purisa S, Tuzun S. Serum Des-GammaCarboxyprothrombin in Patients With Pancreatic Head Adenocarcinoma. Bratisl Lek Listy (2011) 112:552-4.

28. Yamanaka R, Sekino Y, Babasaki T, Kitano H, Ikeda K, Goto K, et al. Renal Metastasis From Primary Hepatocellular Carcinoma: A Case Report. Int Cancer Conf J (2020) 9:141-5. doi: 10.1007/s13691-020-00409-3

29. Pozzi N, Chen Z, Gohara DW, Niu W, Heyduk T, Di Cera E. Crystal Structure of Prothrombin Reveals Conformational Flexibility and Mechanism of Activation. J Biol Chem (2013) 288:22734-44. doi: 10.1074/ jbc.M113.466946

30. Ganrot PO, Niléhn JE. Plasma Prothrombin During Treatment With Dicumarol. II. Demonstration of an Abnormal Prothrombin Fraction. Scand J Clin Lab Invest (1968) 22:23-8. doi: 10.3109/00365516809160731

31. Uehara S, Gotoh K, Handa H, Honjo K, Hirayama A. Process of Carboxylation of Glutamic Acid Residues in the Gla Domain of Human Des-Gamma-Carboxyprothrombin. Clin Chim Acta (1999) 289:33-44. doi: 10.1016/S0009-8981(99)00152-7

32. Ratcliffe JV, Furie B, Furie BC. The Importance of Specific GammaCarboxyglutamic Acid Residues in Prothrombin. Evaluation by SiteSpecific Mutagenesis. J Biol Chem (1993) 268:24339-45. doi: 10.1016/ S0021-9258(20)80531-6

33. Qi F, Zhou A, Yan L, Yuan X, Wang D, Chang R, et al. The Diagnostic Value of PIVKA-II, AFP, AFP-L3, CEA, and Their Combinations in Primary and Metastatic Hepatocellular Carcinoma. J Clin Lab Anal (2020) 34:e23158. doi: 10.1002/jcla.23158

34. Hemker HC, Veltkamp JJ, Hensen A, Loeliger EA. Nature of Prothrombin Biosynthesis: Preprothrombinaemia in Vitamin K-Deficiency. Nature (1963) 200:589-90. doi: 10.1038/200589a0

35. Niléhn JE, Ganrot PO. Plasma Prothrombin During Treatment With Dicumarol. I. Immunochemical Determination of Its Concentration in Plasma. Scand J Clin Lab Invest (1968) 22:17-22. doi: 10.3109/ 00365516809160730

36. Stenflo J. Vitamin K and the Biosynthesis of Prothrombin. II. Structural Comparison of Normal and Dicoumarol-Induced Bovine Prothrombin. J Biol Chem (1972) 247:8167-75. doi: 10.1016/S0021-9258(20)81824-9

37. Liebman HA, Furie BC, Tong MJ, Blanchard RA, Lo KJ, Lee SD. DesGamma-Carboxy (Abnormal) Prothrombin as a Serum Marker of Primary Hepatocellular Carcinoma. N Engl J Med (1984) 310:1427-31. doi: 10.1056/ NEJM198405313102204

38. Liebman HA. Isolation and Characterization of a Hepatoma-Associated Abnormal (Des-Gamma-Carboxy)Prothrombin. Cancer Res (1989) 49:6493-7.

39. Miyakawa T, Kajiwara Y, Shirahata A, Okamoto K, Itoh H, Ohsato K. Vitamin K Contents in Liver Tissue of Hepatocellular Carcinoma Patients. Jpn J Cancer Res (2000) 91:68-74. doi: 10.1111/j.1349-7006.2000.tb00861.x 
40. Dahlberg S, Ede J, Schött U. Vitamin K and Cancer. Scand J Clin Lab Invest (2017) 77:555-67. doi: 10.1080/00365513.2017.1379090

41. Liska DJ, Suttie JW. Location of Gamma-Carboxyglutamyl Residues in Partially Carboxylated Prothrombin Preparations. Biochemistry (1988) 27:8636-41. doi: 10.1021/bi00423a019

42. Uehara S, Gotoh K, Handa H, Tomita H, Senshuu M. Distribution of the Heterogeneity of Des-Gamma-Carboxyprothrombin in Patients With Hepatocellular Carcinoma. J Gastroenterol Hepatol (2005) 20:1545-52. doi: 10.1111/j.1440-1746.2005.03899.x

43. Davis-Yadley AH, Malafa MP. Vitamins in Pancreatic Cancer: A Review of Underlying Mechanisms and Future Applications. Adv Nutr (Bethesda Md.) (2015) 6:774-802.

44. Matsueda K, Yamamoto H, Yoshida Y, Notohara K. Hepatoid Carcinoma of the Pancreas Producing Protein Induced by Vitamin K Absence or Antagonist II (PIVKA-II) and Alpha-Fetoprotein (AFP). J Gastroenterol (2006) 41:1011-9. doi: 10.1007/s00535-006-1889-8

45. Cui SX, Zhang YS, Chu JH, Song ZY, Qu XJ. Des-Gamma-Carboxy Prothrombin (DCP) Antagonizes the Effects of Gefitinib on Human Hepatocellular Carcinoma Cells. Cell Physiol Biochem (2015) 35:201-12. doi: $10.1159 / 000369688$

46. Bruix J, Sherman M. Management of Hepatocellular Carcinoma: An Update. Hepatology (2011) 53:1020-2. doi: 10.1002/hep.24199

47. Kudo M, Takamine Y, Nakamura K, Shirane H, Uchida H, Kasakura S, et al. Des-Gamma-Carboxy Prothrombin (PIVKA-II) and Alpha-FetoproteinProducing IIc-Type Early Gastric Cancer. Am J Gastroenterol (1992) 87:1859-62.

48. Tartaglione S, Pecorella I, Zarrillo SR, Granato T, Viggiani V, Manganaro L, et al. Protein Induced by Vitamin K Absence II (PIVKA-II) as a Potential Serological Biomarker in Pancreatic Cancer: A Pilot Study. Biochemia Med (2019) 29:020707. doi: 10.11613/BM.2019.020707

49. Gao J, Feng X, Inagaki Y, Song P, Kokudo N, Hasegawa K, et al. Des- $\gamma$ Carboxy Prothrombin and C-Met Were Concurrently and Extensively Expressed in Hepatocellular Carcinoma and Associated With Tumor Recurrence. Bioscience Trends (2012) 6:153-9. doi: 10.5582/bst.2012. v6.4.153

50. Suzuki M, Shiraha H, Fujikawa T, Takaoka N, Ueda N, Nakanishi Y, et al. Des-Gamma-Carboxy Prothrombin Is a Potential Autologous Growth Factor for Hepatocellular Carcinoma. J Biol Chem (2005) 280:6409-15. doi: 10.1074/jbc.M406714200

51. Yue P, Gao Z, Xue X, Cui SX, Zhao CR, Yuan Y, et al. Des- $\gamma$-Carboxyl Prothrombin Induces Matrix Metalloproteinase Activity in Hepatocellular Carcinoma Cells by Involving the ERK1/2 MAPK Signalling Pathway. Eur J Cancer (Oxford Engl 1990) (2011) 47:1115-24. doi: 10.1016/j.ejca.2011. 01.017

52. Zhang Y, Chu JH, Cui SX, Song ZY, Qu XJ. Des- $\gamma$-Carboxy Prothrombin (DCP) as a Potential Autologous Growth Factor for the Development of Hepatocellular Carcinoma. Cell Physiol Biochem Int J Exp Cell Physiol Biochem Pharmacol (2014) 34:903-15. doi: 10.1159/000366308

53. Soriano-Garcia M, Padmanabhan K, de Vos AM, Tulinsky A. The Ca2+ Ion and Membrane Binding Structure of the Gla Domain of Ca-Prothrombin Fragment 1. Biochemistry (1992) 31:2554-66. doi: 10.1021/bi00124a016

54. Wang SB, Cheng YN, Cui SX, Zhong JL, Ward SG, Sun LR, et al. DesGamma-Carboxy Prothrombin Stimulates Human Vascular Endothelial Cell Growth and Migration. Clin Exp Metastasis (2009) 26:469-77. doi: 10.1007/s10585-009-9246-y

55. Fujikawa T, Shiraha H, Ueda N, Takaoka N, Nakanishi Y, Matsuo N, et al. Des-Gamma-Carboxyl Prothrombin-Promoted Vascular Endothelial Cell Proliferation and Migration. J Biol Chem (2007) 282:8741-8. doi: 10.1074/ jbc.M609358200

56. Singal A, Volk M, Waljee A, Salgia R, Higgins P, Rogers MA, et al. MetaAnalysis: Surveillance With Ultrasound for Early-Stage Hepatocellular Carcinoma in Patients With Cirrhosis. Alimentary Pharmacol Ther (2009) 30:37-47. doi: 10.1111/j.1365-2036.2009.04014.x

57. Kokudo N, Takemura N, Hasegawa K, Takayama T, Kubo S, Shimada M, et al. Clinical Practice Guidelines for Hepatocellular Carcinoma: The Japan Society of Hepatology 2017 (4th JSH-HCC Guidelines) 2019 Update. Hepatol Res (2019) 49:1109-13. doi: 10.1111/hepr.13411
58. Noureddin M, Rinella ME. Nonalcoholic Fatty Liver Disease, Diabetes, Obesity, and Hepatocellular Carcinoma. Clin Liver Dis (2015) 19:361-79. doi: 10.1016/j.cld.2015.01.012

59. Miyata T, Hayashi H, Yamashita YI, Matsumura K, Higashi T, Imai K, et al. ASO Visual Abstract: The Impact of Histological Liver Inflammation on Oncology and the Prognosis of Patients Undergoing Hepatectomy for Hepatocellular Carcinoma. Ann Surg Oncol (2021). doi: 10.1245/s10434021-10752-1

60. de Lope CR, Tremosini S, Forner A, Reig M, Bruix J. Management of HCC. J Hepatol (2012) 56 Suppl 1:S75-87. doi: 10.1016/S0168-8278(12)60009-9

61. Afshar M, Fletcher P, Bardoli AD, Ma YT, Punia P. Non-Secretion of AFP and Neutrophil Lymphocyte Ratio as Predictors for Survival in Hepatocellular Carcinoma Patients Treated With Sorafenib: A Large UK Cohort. Oncotarget (2018) 9:16988-95. doi: 10.18632/oncotarget.24769

62. Clinical Practice Guidelines EASL. Management of Chronic Hepatitis B. J Hepatol (2009) 50:227-42. doi: 10.1016/j.jhep.2008.10.001

63. Omata M, Cheng AL, Kokudo N, Kudo M, Lee JM, Jia J, et al. Asia-Pacific Clinical Practice Guidelines on the Management of Hepatocellular Carcinoma: A 2017 Update. Hepatol Int (2017) 11:317-70. doi: 10.1007/ s12072-017-9799-9

64. Chen J, Wu G, Li Y. Evaluation of Serum Des-Gamma-Carboxy Prothrombin for the Diagnosis of Hepatitis B Virus-Related Hepatocellular Carcinoma: A Meta-Analysis. Dis Markers (2018) 2018:8906023. doi: 10.1155/2018/8906023

65. Caviglia G, Ciruolo M, Abate M, Carucci P, Rolle E, Rosso C, et al. AlphaFetoprotein, Protein Induced by Vitamin K Absence or Antagonist II and Glypican-3 for the Detection and Prediction of Hepatocellular Carcinoma in Patients With Cirrhosis of Viral Etiology. Cancers (2020) 12(11):3218. doi: $10.3390 /$ cancers 12113218

66. Keller L, Pantel K. Unravelling Tumour Heterogeneity by Single-Cell Profiling of Circulating Tumour Cells. Nat Rev Cancer (2019) 19:553-67. doi: 10.1038/s41568-019-0180-2

67. Tapper EB, Lok AS. Use of Liver Imaging and Biopsy in Clinical Practice. N Engl J Med (2017) 377:756-68. doi: 10.1056/NEJMra1610570

68. Ricco G, Cosma C, Bedogni G, Biasiolo A, Guarino M, Pontisso P, et al. Modeling the Time-Related Fluctuations of AFP and PIVKA-II Serum Levels in Patients With Cirrhosis Undergoing Surveillance for Hepatocellular Carcinoma. Cancer Biomark (2020) 29:189-96. doi: 10.3233/CBM-190118

69. Liu C, Yang S, Wang K, Bao X, Liu Y, Zhou S, et al. Alkaloids From Traditional Chinese Medicine Against Hepatocellular Carcinoma. Biomed Pharmacotherapy = Biomed Pharmacotherapie (2019) 120:109543. doi: 10.1016/j.biopha.2019.109543

70. Feng H, Li B, Li Z, Wei Q, Ren L. PIVKA-II Serves as a Potential Biomarker That Complements AFP for the Diagnosis of Hepatocellular Carcinoma. BMC Cancer (2021) 21:401. doi: 10.1186/s12885-021-08138-3

71. Li T, Yu Y, Liu J, Tian X, Kong M, Wu L, et al. PIVKA-II Level Is Correlated to Development of Portal Vein Tumor Thrombus in Patients With HBVRelated Hepatocellular Carcinoma. Infect Agents Cancer (2019) 14:13. doi: 10.1186/s13027-019-0229-6

72. Shimada M, Yamashita Y, Hamatsu T, Hasegawa H, Utsunomiya T, Aishima $\mathrm{S}$, et al. The Role of Des-Gamma-Carboxy Prothrombin Levels in Hepatocellular Carcinoma and Liver Tissues. Cancer Lett (2000) 159:8794. doi: 10.1016/S0304-3835(00)00539-5

73. Tarao K, Nozaki A, Komatsu H, Komatsu T, Taguri M, Tanaka K, et al. Real Impact of Tumor Marker AFP and PIVKA-II in Detecting Very Small Hepatocellular Carcinoma ( $\leq 2 \mathrm{Cm}$, Barcelona Stage 0) - Assessment With Large Number of Cases. World J Hepatol (2020) 12:1046-54. doi: 10.4254/ wjh.v12.i11.1046

74. Kato K, Iwasaki Y, Taniguchi M, Onodera K, Matsuda M, Kawakami T, et al. Primary Colon Cancer With a High Serum PIVKA-II Level. Int J Surg Case Rep (2015), 95-9. doi: 10.1016/j.ijscr.2014.11.072

75. Svobodova S, Karlikova M, Topolcan O, Pecen L, Pestova M, Kott O, et al. PIVKA-II as a Potential New Biomarker for Hepatocellular Carcinoma - A Pilot Study. In Vivo (Athens Greece) (2018) 32:1551-4. doi: 10.21873/ invivo. 11413

76. Loglio A, Iavarone M, Facchetti F, Di Paolo D, Perbellini R, Lunghi G, et al. The Combination of PIVKA-II and AFP Improves the Detection Accuracy 
for HCC in HBV Caucasian Cirrhotics on Long-Term Oral Therapy. Liver Int Off J Int Assoc Study Liver (2020) 40:1987-96.

77. Yu R, Xiang X, Tan Z, Zhou Y, Wang H, Deng G. Efficacy of PIVKA-II in Prediction and Early Detection of Hepatocellular Carcinoma: A Nested Case-Control Study in Chinese Patients. Sci Rep (2016) 6:35050. doi: 10.1038/srep35050

78. Lim TS, Kim DY, Han KH, Kim HS, Shin SH, Jung KS, et al. Combined Use of AFP, PIVKA-II, and AFP-L3 as Tumor Markers Enhances Diagnostic Accuracy for Hepatocellular Carcinoma in Cirrhotic Patients. Scand J Gastroenterol Scand J Gastroentero (2016) 51:344-53. doi: 10.3109/ 00365521.2015.1082190

79. Sultanik P, Ginguay A, Vandame J, Popovici T, Meritet JF, Cynober L, et al. Diagnostic Accuracy of Des-Gamma-Carboxy Prothrombin for Hepatocellular Carcinoma in a French Cohort Using the Lumipulse $\left({ }^{\circledR}\right)$ G600 Analyzer. J Viral Hepat (2017) 24:80-5. doi: 10.1111/jvh.12622

80. Hemken P, Sokoll L, Yang X, Dai J, Elliott D, Gawel SH, et al. Validation of a Novel Model for the Early Detection of Hepatocellular Carcinoma. Clin Proteomics (2019) 16:2. doi: 10.1186/s12014-018-9222-0

81. Yang T, Xing H, Wang G, Wang N, Liu M, Yan C, et al. A Novel Online Calculator Based on Serum Biomarkers to Detect Hepatocellular Carcinoma Among Patients With Hepatitis B. Clin Chem (2019) 65:1543-53. doi: 10.1373/clinchem.2019.308965

82. Best J, Bilgi H, Heider D, Schotten C, Manka P, Bedreli S, et al. The GALAD Scoring Algorithm Based on AFP, AFP-L3, and DCP Significantly Improves Detection of BCLC Early Stage Hepatocellular Carcinoma. Z Fur Gastroenterologie (2016) 54:1296-305. doi: 10.1055/s-0042-119529

83. Basile U, Miele L, Napodano C, Ciasca G, Gulli F, Pocino K, et al. The Diagnostic Performance of PIVKA-II in Metabolic and Viral Hepatocellular Carcinoma: A Pilot Study. Eur Rev Med Pharmacol Sci (2020) 24:12675-85. doi: 10.26355/eurrev_202012_24165

84. Llovet JM, Zucman-Rossi J, Pikarsky E, Sangro B, Schwartz M, Sherman M, et al. Hepatocellular Carcinoma. Nat Rev Dis Primers (2016) 2:16018. doi: 10.1038/nrdp.2016.18

85. Tang A, Hallouch O, Chernyak V, Kamaya A, Sirlin CB. Epidemiology of Hepatocellular Carcinoma: Target Population for Surveillance and Diagnosis. Abdom Radiol (NY) (2018) 43:13-25. doi: 10.1007/s00261-0171209-1

86. Payancé A, Dioguardi Burgio M, Peoc'h K, Achahboun M, Albuquerque M, Devictor J, et al. Biological Response Under Treatment and Prognostic Value of Protein Induced by Vitamin K Absence or Antagonist-II in a French Cohort of Patients With Hepatocellular Carcinoma. Eur J Gastroenterol Hepatol (2020) 32:1364-72. doi: 10.1097/MEG.0000000000001652

87. Sultanik P, Ginguay A, Vandame J, Popovici T, Meritet JF, Cynober L, et al. Diagnostic Accuracy of Des-Gamma-Carboxy Prothrombin for Hepatocellular Carcinoma in a French Cohort Using the Lumipulse G600 Analyzer. J Viral Hepatitis (2017) 24:80-5. doi: 10.1111/jvh.12622

88. Bruix J, Gores GJ, Mazzaferro V. Hepatocellular Carcinoma: Clinical Frontiers and Perspectives. Gut (2014) 63:844-55. doi: 10.1136/gutjnl2013-306627

89. Dai CY, Yeh ML, Yu ML. An EASL Position Paper for Systemic Treatment of Hepatocellular Carcinoma: Go Forward Courageously. J Hepatol (2021). doi: 10.1016/j.jhep.2021.08.023

90. Poté N, Cauchy F, Albuquerque M, Cros J, Soubrane O, Bedossa P, et al. Contribution of Virtual Biopsy to the Screening of Microvascular Invasion in Hepatocellular Carcinoma: A Pilot Study. Liver Int Off J Int Assoc Study Liver (2018) 38:687-94. doi: 10.1111/liv.13585

91. Lee HW, Song GW, Lee SG, Kim JM, Joh JW, Han DH, et al. Patient Selection by Tumor Markers in Liver Transplantation for Advanced Hepatocellular Carcinoma. Liver Transplant Off Publ Am Assoc Study Liver Dis Int Liver Transplant Soc (2018) 24:1243-51. doi: 10.1002/lt.25056

92. Lai Q, Iesari S, Levi Sandri GB, Lerut J. Des-Gamma-Carboxy Prothrombin in Hepatocellular Cancer Patients Waiting for Liver Transplant: A Systematic Review and Meta-Analysis. Int J Biol Markers (2017) 32:e3704. doi: 10.5301/ijbm.5000276

93. He Y, He K, Huang RQ, Liu LW, Ye SW, Qian JL, et al. A Clinical Scoring System for Predicting Tumor Recurrence After Percutaneous Radiofrequency Ablation for $3 \mathrm{Cm}$ or Less Hepatocellular Carcinoma. Sci Rep (2021) 11:8275. doi: 10.1038/s41598-021-87782-y
94. Kim CG, Lee HW, Choi HJ, Lee JI, Lee HW, Kim SU, et al. Development and Validation of a Prognostic Model for Patients With Hepatocellular Carcinoma Undergoing Radiofrequency Ablation. Cancer Med (2019) 8:5023-32. doi: 10.1002/cam4.2417

95. Kim H, Lee S, Yoon M. Alpha-Fetoprotein Is Correlated With Intrahepatic Recurrence of Hepatocellular Carcinoma After a Hepatectomy. Ann Surg Treat Res (2020) 98:168-76. doi: 10.4174/astr.2020.98.4.168

96. Lim T, Rhee H, Kim GM, Kim SU, Kim BK, Park JY, et al. AlphaFetoprotein, Des-Gamma-Carboxy Prothrombin, and Modified RECIST Response as Predictors of Survival After Transarterial Radioembolization for Hepatocellular Carcinoma. J Vasc Interventional Radiol JVIR (2019) 30:1194-1200.e1. doi: 10.1016/j.jvir.2019.03.016

97. Ichikawa T, Machida N, Sasaki H, Tenmoku A, Kaneko H, Negishi R, et al. Early Prediction of the Outcome Using Tumor Markers and mRECIST in Unresectable Hepatocellular Carcinoma Patients Who Underwent Transarterial Chemoembolization. Oncology (2016) 91:317-30. doi: $10.1159 / 000448999$

98. Kulik L, El-Serag HB. Epidemiology and Management of Hepatocellular Carcinoma. Gastroenterology (2019) 156:477-91.el. doi: 10.1053/j.gastro. 2018.08.065

99. Yanagaki M, Haruki K, Yasuda J, Furukawa K, Onda S, Tsunematsu M, et al. The Significance of the Rapid Turnover Protein Score as a Predictor of the Long-Term Outcomes in Hepatocellular Carcinoma After Hepatic Resection. Ann Surg Oncol (2021). doi: 10.1245/s10434-021-10723-6

100. Toyoda H, Kumada T, Osaki Y, Oka H, Urano F, Kudo M, et al. Staging Hepatocellular Carcinoma by a Novel Scoring System (BALAD Score) Based on Serum Markers. Clin Gastroenterol Hepatol (2006) 4:1528-36. doi: 10.1016/j.cgh.2006.09.021

101. Fox R, Berhane S, Teng M, Cox T, Tada T, Toyoda H, et al. Biomarker-Based Prognosis in Hepatocellular Carcinoma: Validation and Extension of the BALAD Model. Br J Cancer (2014) 110:2090-8. doi: 10.1038/bjc.2014.130

102. Li B, Zhao Y, Cai W, Ming A, Li H. Validation and Update of a Multivariable Prediction Model for the Identification and Management of Patients at Risk for Hepatocellular Carcinoma. Clin Proteomics (2021) 18:21. doi: 10.1186/ s12014-021-09326-w

103. Wongjarupong N, Negron-Ocasio GM, Chaiteerakij R, Addissie BD, Mohamed EA, Mara KC, et al. Model Combining Pre-Transplant Tumor Biomarkers and Tumor Size Shows More Utility in Predicting Hepatocellular Carcinoma Recurrence and Survival Than the BALAD Models. World J Gastroenterol (2018) 24:1321-31. doi: 10.3748/wjg.v24.i12.1321

104. Wang X, Zhang W, Liu Y, Gong W, Sun P, Kong X, et al. Diagnostic Value of Prothrombin Induced by the Absence of Vitamin K or Antagonist-II (PIVKA-II) for Early Stage HBV Related Hepatocellular Carcinoma. Infect Agent Cancer (2017) 12:47. doi: 10.1186/s13027-017-0153-6

105. Okamura Y, Sugiura T, Ito T, Yamamoto Y, Ashida R, Aramaki T, et al. The Predictors of Microscopic Vessel Invasion Differ Between Primary Hepatocellular Carcinoma and Hepatocellular Carcinoma With a Treatment History. World J Surg (2018) 42:3694-704. doi: 10.1007/s00268018-4658-y

106. Wu J, Xiang Z, Bai L, He L, Tan L, Hu M, et al. Diagnostic Value of Serum PIVKA-II Levels for BCLC Early Hepatocellular Carcinoma and Correlation With HBV DNA. Cancer Biomarkers Section A Dis Markers (2018) 23:23542. doi: $10.3233 / \mathrm{CBM}-181402$

107. Ryu T, Takami Y, Wada Y, Tateishi M, Hara T, Yoshitomi M, et al. A Clinical Scoring System for Predicting Microvascular Invasion in Patients With Hepatocellular Carcinoma Within the Milan Criteria. J Gastrointestinal Surg Off J Soc Surg Alimentary Tract (2019) 23:779-87. doi: 10.1007/ s11605-019-04134-y

108. Si YQ, Wang XQ, Fan G, Wang CY, Zheng YW, Song X, et al. Value of AFP and PIVKA-II in Diagnosis of HBV-Related Hepatocellular Carcinoma and Prediction of Vascular Invasion and Tumor Differentiation. Infect Agents Cancer (2020) 15:70. doi: 10.1186/s13027-020-00337-0

109. Fernlund P, Stenflo J, Roepstorff P, Thomsen J. Vitamin K and the Biosynthesis of Prothrombin. V. Gamma-Carboxyglutamic Acids, the Vitamin K-Dependent Structures in Prothrombin. J Biol Chem (1975) 250:6125-33. doi: 10.1016/S0021-9258(19)41168-X

110. Okuda H, Nakanishi T, Takatsu K, Saito A, Hayashi N, Yamamoto M, et al. Comparison of Clinicopathological Features of Patients With Hepatocellular 
Carcinoma Seropositive for Alpha-Fetoprotein Alone and Those Seropositive for Des-Gamma-Carboxy Prothrombin Alone. J Gastroenterol Hepatol (2001) 16:1290-6. doi: 10.1046/j.1440-1746.2001.02610.x

111. Ma X, Zhu J, Wu J, Tian L, Gao YY, Zhang CY, et al. Significance of PIVKAII Levels for Predicting Microvascular Invasion and Tumor Cell Proliferation in Chinese Patients With Hepatitis B Virus-Associated Hepatocellular Carcinoma. Oncol Lett (2018) 15:8396-404. doi: 10.3892/ol.2018.8375

112. Suh S, Choi Y. Predictors of Micrometastases in Patients With Barcelona Clinic Liver Cancer Classification B Hepatocellular Carcinoma. Yonsei Med J (2017) 58:737-42. doi: 10.3349/ymj.2017.58.4.737

113. Tsugawa D, Fukumoto T, Kido M, Takebe A, Tanaka M, Kuramitsu K, et al. The Predictive Power of Serum $\alpha$-Fetoprotein and Des- $\gamma$-Carboxy Prothrombin for Survival Varies by Tumor Size in Hepatocellular Carcinoma. Kobe J Med Sci (2015) 61:E124-31.

114. Kim J, Kwon C, Joh JW, Yoo H, Kim K, Sinn DH, et al. Nomograms in Hepatectomy Patients With Hepatitis B Virus-Related Hepatocellular Carcinoma. J Gastrointestinal Surg: Off J Soc Surg Alimentary Tract (2019) 23:1559-67. doi: 10.1007/s11605-018-04074-z

115. Shimoda M, Tago K, Shiraki T, Mori S, Kato M, Aoki T, et al. Risk Factors for Early Recurrence of Single Lesion Hepatocellular Carcinoma After Curative Resection. World J Surg (2016) 40:2466-71. doi: 10.1007/s00268-016-3529-7

116. Shimada S, Kamiyama T, Orimo T, Nagatsu A, Asahi Y, Sakamoto Y, et al. Prognoses, Outcomes, and Clinicopathological Characteristics of Very Elderly Patients With Hepatocellular Carcinoma Who Underwent Hepatectomy. World J Surg Oncol (2020) 18:122. doi: 10.1186/s12957-020-01899-4
117. Kudo A, Shinoda M, Ariizumi S, Kumamoto T, Katayama M, Otsubo T, et al. Des-Gamma-Carboxy Prothrombin Affects the Survival of HCC Patients With Marginal Liver Function and Curative Treatment: Acros1402. J Cancer Res Clin Oncol (2020) 146:2949-56. doi: 10.1007/s00432-020-03270-2

118. Zhang ZS, Yang RH, Yao X, Cheng YY, Shi HX, Yao CY, et al. HGF/c-MET Pathway Contributes to Cisplatin-Mediated PD-L1 Expression in Hepatocellular Carcinoma. Cell Biol Int (2021). doi: 10.1002/cbin.11697

Conflict of Interest: The authors declare that the research was conducted in the absence of any commercial or financial relationships that could be construed as a potential conflict of interest.

Publisher's Note: All claims expressed in this article are solely those of the authors and do not necessarily represent those of their affiliated organizations, or those of the publisher, the editors and the reviewers. Any product that may be evaluated in this article, or claim that may be made by its manufacturer, is not guaranteed or endorsed by the publisher.

Copyright (c) 2021 Yang, Li, Lu, Liu, Kong and Liu. This is an open-access article distributed under the terms of the Creative Commons Attribution License (CC BY). The use, distribution or reproduction in other forums is permitted, provided the original author(s) and the copyright owner(s) are credited and that the original publication in this journal is cited, in accordance with accepted academic practice. No use, distribution or reproduction is permitted which does not comply with these terms. 